\title{
A Spectral Approach to Acoustic Absorption Measurement
}

\author{
Leander Claes ${ }^{1}$, René Spencer Chatwell ${ }^{2}$, Jadran Vrabec ${ }^{2}$, Bernd Henning ${ }^{1}$ \\ 1 Measurement Engineering Group, Paderborn University, Warburger Str. 100, \\ 33098 Paderborn, Germany, \\ claes@emt.uni-paderborn.de \\ 2 Thermodynamics and Energy Technology, Paderborn University, Warburger Str. 100, \\ 33098 Paderborn, Germany
}

\begin{abstract}
:
Next to the sound velocity, the absorption coefficient of acoustic waves in fluids is an important thermodynamic property. Although measurements of acoustic absorption have been conducted in the past, most of them negate the influence of parasitic dissipative effects or rely on either analytical or empirical compensation. A typical method for measuring acoustic absorption is to determine the decline of the acoustic signal amplitude as the wave propagates through the medium. We present an alternative approach, by utilizing that acoustic waves experience dispersive effects in absorbing media. It is known that acoustic absorption in fluids increases with the squared frequency of the acoustic wave. On this basis, we deduce an analytic expression that links the decline of the spectral centroid (center frequency) of the acoustic signal to the medium's absorption coefficient. The results are verified using a simulation environment that is based on finite differences.
\end{abstract}

Key words: absorption, fluids, dispersive effects, center frequency, spectral centroid

\section{Motivation}

High precision measurements of all parameters are necessary for a complete thermodynamic description of a fluid. Among others, these are the sound velocity $c$, the density $\rho$, the shear viscosity $\mu_{\mathrm{S}}$, the bulk viscosity $\mu_{\mathrm{B}}$, the isobaric and isochoric molar heat capacities $C_{\mathrm{P}}$ and $C_{\mathrm{V}}$, and the thermal conductivity $\lambda$. In the case of linear acoustics, these parameters cause absorption, according to the following relation [6-8]:

$$
\alpha=\frac{\omega^{2}}{2 \rho c^{3}}\left(\frac{4}{3} \mu_{\mathrm{S}}+\mu_{\mathrm{B}}+\frac{C_{\mathrm{P}}-C_{\mathrm{V}}}{C_{\mathrm{P}} \cdot C_{\mathrm{V}}} \lambda\right),
$$

where $\omega$ is the angular frequency of the acoustic wave. While the other parameters are well known, there is still only fragmentary knowledge of the bulk viscosity $\mu_{\mathrm{B}}$ for most fluids. By precise measurements of acoustic absorption, equation (1) allows to determine the bulk viscosity, given all other parameters are known. There are numerous technical applications in fluid analysis that would benefit from a more precise measurement method for acoustic absorption.

The classical approach to measure absorption is to analyze the acoustic wave amplitude's decay as it travels through the medium. Several arrangements of transducers and reflectors can be employed to realize such a measurement. In [1], a transmission line setup with a sending and a receiving transducer is used. In contrast, the work presented in [5] applies a single quartz transducer and a reflecting surface.

Absorption constitutes only one mechanism to affect an acoustic wave's amplitude, while diffraction, i.e. the widening of the acoustic burst, caused by the source's finite dimensions, is another. There are options to compensate for diffraction [5], yet they typically rely on assumptions about the transducer (i.e. a pistontype oscillator) that cannot be guaranteed for most physical measurement setups. To avoid acoustic near field effects, measurements should be performed in the far field, enabled by increasing the acoustic transmission distance, decreasing the transducer's diameter or lowering the excitation frequency. In the acoustic far field, the signal amplitude decreases almost reciprocally with distance [3]. However, this is often hard to realize due to limitations in the apparatus' geometry [9].

Here, we propose an approach to attain the absorption coefficient $\alpha$ by analyzing the spectral distribution of a received ultrasonic burst signal.

\section{Theoretical considerations}

The mechanism, by which acoustic waves are absorbed, implies that higher frequencies yield greater attenuation (cf. equation (1)). This leads to a change of the acoustic wave's spectral 
distribution. Further, the absorption coefficient $\alpha$ is expressed as the reduced absorption coefficient $\check{\alpha}$ multiplied by the squared angular frequency:

$$
\alpha(\omega)=\check{\alpha} \cdot \omega^{2} .
$$

It is assumed that an ultrasonic transducer emits an acoustic wave with a spectral distribution $U_{0}(\omega)$, which allows to model its change by using an exponential decay function:

$$
\begin{gathered}
U(\omega, z)=d(z) \cdot U_{0}(\omega) \mathrm{e}^{-\alpha(\omega) \cdot \mathrm{z}}, \\
=d(z) \cdot U_{0}(\omega) \mathrm{e}^{-\check{\alpha} \omega^{2} \cdot \mathrm{z}},
\end{gathered}
$$

where $z$ is the propagation distance of the acoustic wave and $d(z)$ is a frequencyindependent, but yet unspecified damping factor. We will consider the centroid of this spectral distribution $U(\omega, z)$ similar to [4] and [10]. While Quam et al. [4] applied this method to seismic waves; Fink et al. [10] used it to estimate attenuation in liver tissue.

The centroid of the spectral distribution or spectral centroid (angular center frequency) $\omega_{\mathrm{C}}(z)$ is defined as:

$$
\omega_{\mathrm{C}}(z)=\frac{m_{1, U}(z)}{m_{0, U}(z)},
$$

where $m_{n, U}$ is the $n$-th raw moment of the spectral distribution $U(\omega, z)$, given by:

$$
m_{n, U}(z)=\int_{-\infty}^{\infty} \omega^{n} U(\omega, z) \mathrm{d} \omega .
$$

Inserting equation (5) into (4) yields:

$$
\begin{aligned}
& \omega_{\mathrm{C}}(z)=\frac{\int_{-\infty}^{\infty} \omega \cdot U(\omega, z) \mathrm{d} \omega}{\int_{-\infty}^{\infty} U(\omega, z) \mathrm{d} \omega}, \\
& =\frac{\int_{-\infty}^{\infty} \omega \cdot d(z) \cdot U_{0}(\omega) \mathrm{e}^{-\breve{\alpha} \omega^{2} \cdot \mathrm{z}} \mathrm{d} \omega}{\int_{-\infty}^{\infty} d(z) \cdot U_{0}(\omega) \mathrm{e}^{-\breve{\alpha} \omega^{2} \cdot \mathrm{z}} \mathrm{d} \omega}, \\
& =\frac{\int_{-\infty}^{\infty} \omega \cdot U_{0}(\omega) \mathrm{e}^{-\breve{\alpha} \omega^{2} \cdot \mathrm{z}} \mathrm{d} \omega}{\int_{-\infty}^{\infty} U_{0}(\omega) \mathrm{e}^{-\breve{\alpha} \omega^{2} \cdot \mathrm{z}} \mathrm{d} \omega} .
\end{aligned}
$$

The fact that $d(z)$ can be eliminated from the equation demonstrates that the observed spectral centroid is not a function of the frequency-independent damping mechanisms. For further analysis, we compute the spectral centroid's $\omega_{\mathrm{C}}(z)$ spatial derivative. To do so, we have to calculate the spatial derivative of the raw moments first, omitting $d(z)$, as shown in equation (6):

$$
\begin{gathered}
\frac{\partial m_{n, U}(z)}{\partial z} \\
=\int_{-\infty}^{\infty} \omega^{n} U_{0}(\omega) \mathrm{e}^{-\breve{\alpha} \omega^{2} \cdot z} \cdot\left(-\check{\alpha} \omega^{2}\right) \mathrm{d} \omega,
\end{gathered}
$$

$$
\begin{gathered}
=-\check{\alpha} \int_{-\infty}^{\infty} \omega^{n+2} U_{0}(\omega) \mathrm{e}^{-\check{\alpha} \omega^{2} \cdot \mathrm{z}} \mathrm{d} \omega, \\
=-\check{\alpha} \cdot m_{n+2, U} .
\end{gathered}
$$

This expression allows computing the anticipated spectral centroid's spatial derivative:

$$
\begin{gathered}
\frac{\partial \omega_{\mathrm{C}}(z)}{\partial z} \\
=\frac{-\check{\alpha}\left(m_{3, U}(z) m_{0, U}(z)-m_{2, U}(z) m_{1, U}(z)\right)}{\left(m_{0, U}(z)\right)^{2}}, \\
=\check{\alpha} \frac{\left(m_{2, U}(z) m_{1, U}(z)-m_{3, U}(z) m_{0, U}(z)\right)}{\left(m_{0, U}(z)\right)^{2}} .
\end{gathered}
$$

In order to calculate the reduced absorption coefficient $\breve{\alpha}$, the first four raw moments $\left(m_{0, U}(z)\right.$ to $\left.m_{3, U}(z)\right)$ of the signal's spectral distribution and the spectral centroid's spatial derivative need to be known:

$$
\begin{gathered}
\check{\alpha}=\frac{\partial \omega_{\mathrm{C}}(z)}{\partial z} \\
\cdot \frac{\left(m_{0, U}(z)\right)^{2}}{\left(m_{2, U}(z) m_{1, U}(z)-m_{3, U}(z) m_{0, U}(z)\right)} .
\end{gathered}
$$

A discretization of equation (5) allows calculating the raw moments of a measured spectral distribution effortlessly, while determining the spectral centroid's slope is more challenging. If the acoustic signal's spectral distribution can be measured at two different positions $z_{1}$ and $z_{2}$, the spatial derivative of $\omega_{\mathrm{C}}$ can be approximated by finite differences.

\section{Simulation environment}

To evaluate if the proposed measurement principle is applicable in laboratory experiments, a simulation environment is created that allows using signals from a virtual measurement setup. Here, parasitic influences, for example the limited bandwidth of the receiving transducer, can be neglected. This allows evaluating how well the measurement principle performs under ideal conditions.

The present simulation environment is based on finite differences in the time domain (FDTD) [11]. The procedure, first presented by Yee in 1966, requires a set of discrete field equations. In linear acoustics, these are the equation of continuity [3]

$$
\frac{\partial p}{\partial t}=-\rho c^{2} \operatorname{div} \vec{v},
$$

and a form of the Navier-Stokes equation to account for viscous losses [3]

$$
\rho \frac{\partial \vec{v}}{\partial t}=-\operatorname{grad} p+\mu \nabla^{2} \vec{v} .
$$

Here, $p$ is the acoustic pressure, $\rho$ is the density of the medium, $c$ is the thermodynamic sound 
velocity and $\vec{v}$ the particle velocity. $\mu$ describes the combined dissipative losses of the fluid:

$$
\mu=\frac{4}{3} \mu_{\mathrm{S}}+\mu_{\mathrm{B}}+\frac{C_{\mathrm{P}}-C_{\mathrm{V}}}{C_{\mathrm{P}} \cdot C_{\mathrm{V}}} \lambda .
$$

Note that the adiabatic equation of state in linearized form is already included in equation (10). In order to realize an acoustic field simulation, equations (10) and (11) need to be discretized. A discretization is achieved by placing the pressure and velocity fields' sampling points on staggered grids (Yee grids [11]) in spatial and temporal direction.

The FDTD approach to simulate acoustic wave phenomena is well documented in the literature and has been applied to multiple problems [12-14] and as we intend to use this methodology in the same manner, we refrain from presenting it in greater detail.

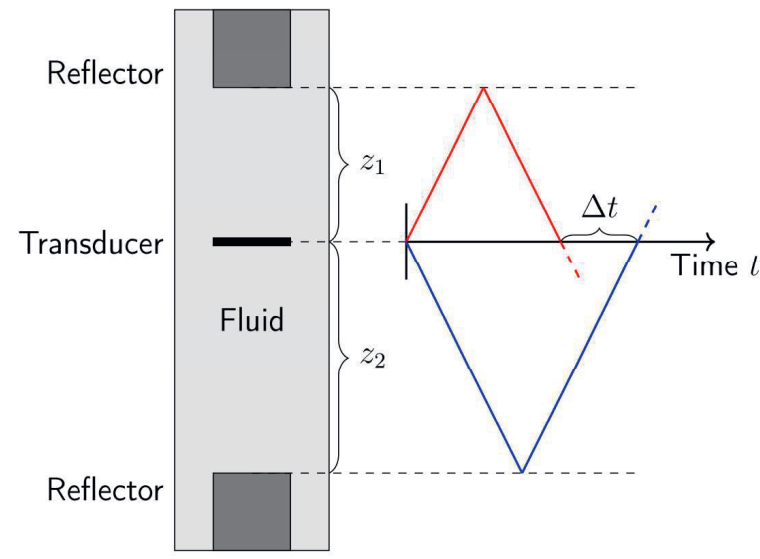

Fig. 1. Schematic of the simulation setup used to generate the signals for the verification of the deduced relation between the shift of the spectral centroid (center frequency) and absorption.

\section{Evaluation of simulated measurement signals}

To apply the present measurement principle for acoustic absorption, we require a measurement setup that allows acquiring a multitude of ultrasound signals, which have propagated different distances from a fixed source. A setup that realizes these criteria consist of a single, suspended quartz disc transducer to allow for sound emission in both directions. Two acoustic reflectors are mounted at different positions from the transducer. They reflect the acoustic wave backwards to the transducer. Due to the differently positioned reflectors, the acoustic bursts that are emitted by the transducer in different directions propagate different distances until they reach the transducer again. Muringer et al. [15] first introduced this setup for precision sound velocity measurements. Figure 1 shows a schematic of the setup.
We apply a one-dimensional simulation environment to model the experimental setup. There are, however, some assumptions made. First, the reflectors are assumed to be ideal. The influence of the transducer on the wave propagation is neglected. Instead, the acoustic pressure is excited directly by a Gaussianmodulated sinusoidal pulse at the transducer position. To acquire the signal, the acoustic pressure at the location of the transducer is recorded. Figure 2 shows such a recorded signal. The distances of the reflectors are $z_{1}=$ $20 \mathrm{~mm}$ and $z_{1}=30 \mathrm{~mm}$, the thermodynamic properties in the simulation are arbitrarily chosen to be $c=1500 \mathrm{~m} / \mathrm{s}, \rho=1000 \mathrm{~kg} / \mathrm{m}^{3}$, and $\mu_{\mathrm{S}}=$ $10 \mathrm{mPa} s$ for proof of concept. Bulk viscosity $\mu_{\mathrm{B}}$ and thermal losses are not present. They could be incorporated in the simulation as well, however, their influence would only be additive to the effect of the shear viscosity $\mu_{\mathrm{S}}$, (cf. equation (1)).

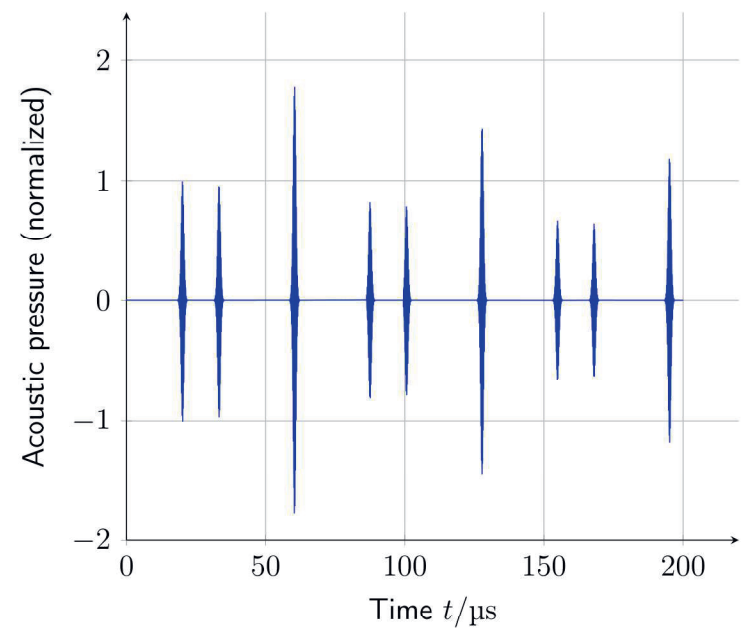

Fig. 2. Signal created by acoustic field simulation of an one-dimensional representation of the measurement setup (figure 1).

As expected, we receive two bursts at about $18 \mu \mathrm{s}$ and $32 \mu \mathrm{s}$. They originated from the acoustic bursts propagated to and reflected at each reflector. As the transducer does not influence the field and is thereby transparent for acoustic waves, each acoustic bust propagates to the opposing reflector. Both bursts had opposing directions, yet they propagated the same distance and consequently interfere constructively at the transducer after about $60 \mu \mathrm{s}$. After the first constructive interference a repeating pattern in the received signals' bursts emerges. The absorption of acoustic energy due to the shear viscosity is visible in the bursts' declining amplitudes.

To apply the proposed measurement procedure to the recorded signal, it is necessary to assign the propagation distance to each burst. The distance propagated by the first two bursts is 
$40 \mathrm{~mm}$ and $60 \mathrm{~mm}$, respectively. The third burst propagated $100 \mathrm{~mm}$, the forth $140 \mathrm{~mm}$, the fifth $160 \mathrm{~mm}$ etc. The spectral centroid of each burst can now be determined by calculating the spectral centroid of its magnitude spectrum. Figure 3 shows the resulting spectral centroids of the distances travelled for simulations with varying shear viscosity $\mu_{\mathrm{S}}$.

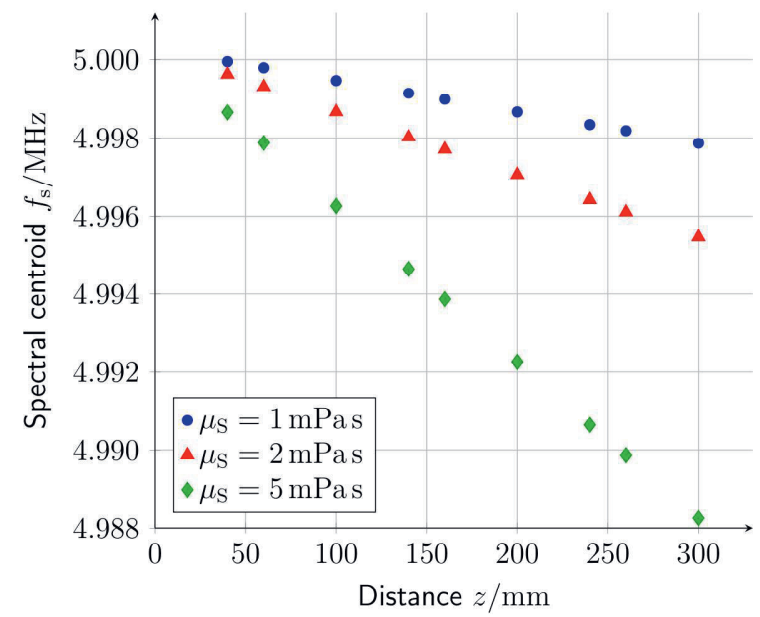

Fig. 3. Spectral centroids of bursts from signals created by acoustic field simulations with the presented simulation environment and varying shear viscosity $\mu_{S}$.

Figure 3 reveals the seemingly linear relationship between the spectral centroid and the burst's propagation distance. The slopes are indicative of the shear viscosity and thereby the dissipative losses in the examined fluid. Mind that this linear relationship was not assumed, yet it facilitates the application of equation (9) to this specific problem. We can easily determine the spectral centroid's spatial derivative by fitting a straight line through the spectral centroids by means of least squares minimization. The slope of this straight line can then be used as an estimate of the aforementioned derivative

$$
\frac{\partial \omega_{\mathrm{C}}(z)}{\partial z} \text {. }
$$

To compute the reduced absorption of the fluid $\check{\alpha}$, the raw moments of the magnitude spectra of the bursts are required. It should be noted, however, that even if the raw moments $m_{n, U}(z)$ of the acoustic burst's magnitude spectra may vary while propagation through the medium, the expression

$$
\frac{\left(m_{0, U}(z)\right)^{2}}{m_{2, U}(z) m_{1, U}(z)-m_{3, U}(z) m_{0, U}(z)},
$$

as well as the spectral centroid's derivative are constant. Evaluating expression (14) for each burst of a recorded signal confirms this assumption to a satisfying degree. To handle deviations, we use the mean value of the results of expression (14) for all bursts of a given signal. The resulting formula is a modified form of equation (9) for discrete values of $z$ :

$$
\begin{gathered}
\check{\alpha}=\frac{\partial \omega_{\mathrm{C}}\left[z_{n}\right]}{\partial z} \\
\frac{1}{N} \sum_{n=1}^{N}\left(\frac{\left(m_{0, U}\left[z_{n}\right]\right)^{2}}{\left(m_{2, U}\left[z_{n}\right] m_{1, U}\left[z_{n}\right]-m_{3, U}\left[z_{n}\right] m_{0, U}\left[z_{n}\right]\right)}\right)
\end{gathered}
$$

We are now able to compute the measured reduced absorption $\breve{\alpha}^{\prime}$ and thereby find values for the measured shear viscosity $\mu_{\mathrm{S}}{ }^{\prime}$, given that no other losses are present in the simulation:

$$
\mu_{\mathrm{S}}^{\prime}=\frac{3}{4} \check{\alpha}^{\prime} c^{3} \rho \text {. }
$$

Note that by considering the reduced absorption $\check{\alpha}$, we avoid using a discrete angular frequency $\omega$. This circumvents errors, as the signals are sufficiently broadband to not be considered of a single frequency, cf. table 1 for a summary.

Tab. 1: Results of the absorption measurement using signals generated by acoustic field simulation.

\begin{tabular}{|c|c|c|c|}
\hline $\begin{array}{c}\text { Shear viscosity in } \\
\text { simulation } \mu_{\mathrm{S}} / \mathrm{mPa} \mathrm{s}\end{array}$ & 1.000 & 2.000 & 5.000 \\
\hline $\begin{array}{c}\text { Measured reduced } \\
\text { absorption } \\
\begin{array}{c}\text { coefficient } \\
\check{\alpha}^{\prime} / 10^{-16} \mathrm{~s}^{2} \mathrm{~m}^{-1}\end{array}\end{array}$ & 1.998 & 3.995 & 9.988 \\
\hline $\begin{array}{c}\text { Measured shear } \\
\text { viscosity } \mu_{\mathrm{S}}^{\prime} / \mathrm{mPa} \mathrm{s}\end{array}$ & 1.011 & 2.023 & 5.056 \\
\hline
\end{tabular}

Based on these results, we are able to estimate the coefficient of attenuation (i.e. shear or bulk viscosity) with a deviation of slightly more than $1 \%$ from simulation data. Using finite difference quotients instead of a straight line to estimate the spectral centroids spatial derivative can further improve the results. However, This would yield one point less than the number of measured bursts, as well as values for distances that are located in between the positions of recorded bursts. We therefore propose using central difference quotients and thereby omitting the first and last burst. Expression (14) can thus be evaluated for each burst and multiplied by the values gained by estimating expression (13) with central difference quotients. This results in values for the reduced absorption coefficient $\check{\alpha}^{\prime}$ for each burst (excluding the first and last one). Ideally, these values should be identical, however, for applications deviations are to be expected. Here, one could use an average of these values. This yields a further increase in accuracy with a deviation of about $1.01 \%$ of the simulation. Mind that all deviations, observed in the procedure thus far, overestimate absorption by a small margin. 


\section{Measurement}

For a principal verification of the proposed measurement method, we apply a simple setup, as shown in figure 4. An ultrasound transducer (Olympus K.K., model C306-SU) is placed at the bottom of a tank filled with water. Using a hydrophone (RP acoustics e.K., model RP $35 \mathrm{~s}$ ) mounted on a linear actuator, signals are recorded at different distances from the transducer. The received signals are amplified by a broadband amplifier (FEMTO Messtechnik $\mathrm{GmbH}$, model HVA-10M-60-F). A USBOscilloscope (TiePie, model Handyscope HS5) is used for signal generation and acquisition. The transmitted signal is chosen to be a Gaussian modulated sinusoidal pulse with a center frequency of $2 \mathrm{MHz}$ and a relative bandwidth of 0.1 .

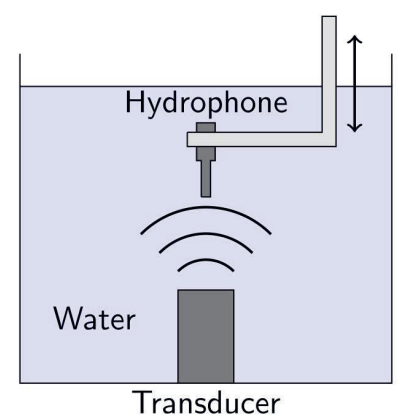

Fig. 4: Measurement setup used to experimentally verify the proposed measurement method.

Figure 5 shows the spectral centroid of the transmitted signal with respect to the distance from the transducer. Very close to the transducer, no clear tendency is visible. However, at distances greater than $30 \mathrm{~mm}$ the expected linear trend emerges. Using the approximation for the near field length $n$ [3],

$$
n=\frac{d_{\mathrm{eff}}^{2}-\lambda^{2}}{4 \lambda},
$$

with the effective diameter of the transducer $d_{\text {eff }}=13 \mathrm{~mm}$ and a wavelength $\lambda=0.745 \mathrm{~mm}$, we estimate the near field to end at a distance of $56.5 \mathrm{~mm}$. The erratic behavior in the vicinity of the transducer can be accounted to the measurement being carried out in the transducer's near field.

While the slope of the linear trend is in the expected regime, evaluating expression (14) does not yield satisfactory results. This may be due to nonlinear effects in the experimental setup that are as of now unaccounted for in the computation but where observed in the recorded signals. For future research, we aim to eliminate these nonlinear effects by adapting the experimental setup or alternatively account for them in the computation.

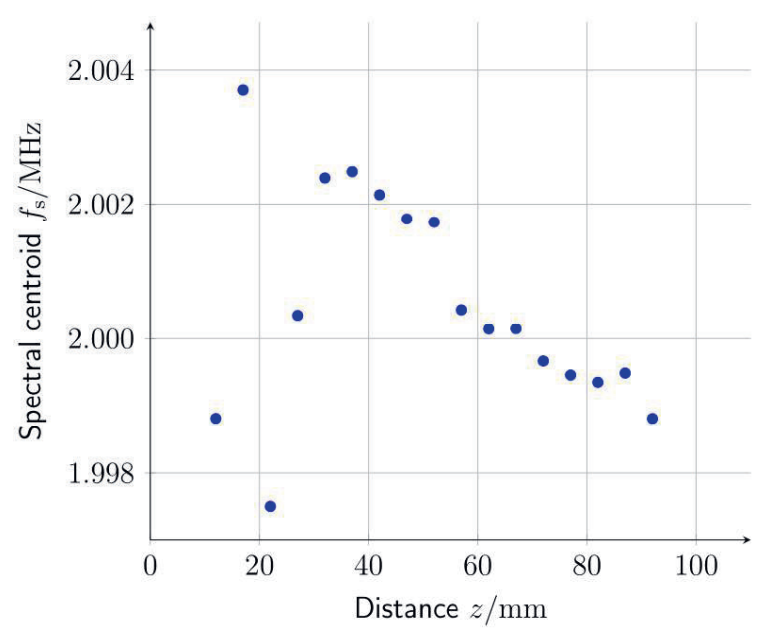

Fig. 5. Spectral centroids of an acoustic burst evaluated at different distances from the transducer in water $\left(\right.$ at $\left.20^{\circ} \mathrm{C}\right)$.

\section{Potential Applications}

Many applications are realizable, since the measurement procedure requires just the transmission of ultrasonic waves through a medium within a vessel of a given length. However, as of now, precise measurements are not possible and require further investigation. Approximate or referenced measurement procedures, however, can be implemented in existing ultrasonic systems. For example in ultrasonic flow or fluid level measurement systems, evaluating the received signal's spectral centroid (center frequency) yields information about absorption in a fluid. The sensitivity of such a measurement increases with the bandwidth of the transmitted signal. If only one received burst is available, as for example in level measurement systems, and multiple reflections are not evaluable, the spectral centroid of the originally transmitted electrical burst can be used as reference for the expected change in the fluid's spectral centroid during transmission of the wave.

\section{Conclusions}

We present a method to estimate ultrasonic absorption in fluids, by evaluating the spectral properties of received signals, i.e. the signals' spectral centroid (center frequency) using ultrasonic transmission measurements. The relation between the statistical properties of the received spectrum and absorption, as derived herein, demonstrate to be a viable concept for determining ultrasonic absorption coefficients of fluids. Although the performed acoustic field simulations obtained good results, the application in a practical measurement system faces challenges that will have to be overcome. The proposed procedure yields results for a qualitative measurement, which we believe is 
sufficiently precise for referenced measurements and industrial applications.

\section{References}

[1] A.S. Dukhin, P.J. Goetz, Bulk viscosity and compressibility measurement using acoustic spectroscopy, The Journal of Chemical Physics 130 (12), 124519 (2009); doi: 10.1063/1.3095471

[2] R. Kuc, M. Schwartz, Estimating the Acoustic Attenuation Coefficient Slope for Liver from Reflected Ultrasound Signals, IEEE Transactions on Sonics and Ultrasonics 26 (5), 353-361 (1979); doi: 10.1109/T-SU.1979.31116

[3] R. Lerch, G.M. Sessler, D. Wolf, Technische Akustik (2009); doi: 10.1007/978-3-540-49833-9

[4] Y. Quan, J.M. Harris, Seismic attenuation tomography using the frequency shift method, Geophysics 62 (3), 895-905 (1997); doi: 10.1190/1.1444197

[5] H. Seki, A. Granato, R. Truell, Diffraction Effects in the Ultrasonic Field of a Piston Source and Their Importance in the Accurate Measurement of Attenuation, The Journal of the Acoustical Society of America 28 (2), 230-238 (1956); doi: 10.1121/1.1908249

[6] P.M. Morse, K.U. Ingard, Theoretical acoustics (1968); doi: 10.1126/science.170.3954.156-a

[7] V.A. Sutilov, Physik des Ultraschalls (1984); doi: 10.1007/978-3-7091-8750-0

[8] M. Trusler, Physical Acoustics and Metrology of Fluids (1991); doi: 10.1604/9780750309400

[9] F.H. Dubberke, D.B. Rasche, E. Baumhögger, J. Vrabec, Apparatus for the measurement of the speed of sound of ammonia up to high temperatures and pressures, Review of Scientific Instruments 85, 084901 (2014); doi: $10.1063 / 1.4891795$

[10] M. Fink, F. Hottier, J.F. Cardoso, Ultrasonic Signal Processing for in Vivo Attenuation Measurement. Short Time Fourier Analysis. Ultrasonic Imaging 5 (2), 117-135 (1983); doi: $10.1177 / 016173468300500202$

[11] K. Yee, Numerical solution of initial boundary value problems involving maxwell's equations in isotropic media, IEEE Trans. Antennas Propagat. 14 (3), S. 302-307 (1966); doi: 10.1109/TAP.1966.1138693

[12] D. Botteldooren, Finite-difference time-domain simulation of low-frequency room acoustic problems, The Journal of the Acoustical Society of America 98 (6), 3302-3308 (1995); doi: $10.1121 / 1.413817$

[13] F.D. Hastings, J.B. Schneider, S.L. Broschat, A finite-difference time-domain solution to scattering from a rough pressure-release surface, The Journal of the Acoustical Society of America 102 (6), 3394-3400 (1997); doi: $10.1121 / 1.419581$
[14] N. Jiménez, J. Redondo, V. Sánchez-Morcillo, F. Camarena, Y. Hou, E.E. Konofagou, Nonlinear Acoustics FDTD method including Frequency Power Law Attenuation for Soft Tissue Modeling, (2014) online: http://arxiv.org/pdf/1401.6669.

[15] M.J.P. Muringer, N.J. Trappeniers, S.N.Biswas, The effect of pressure on the sound velocity and density of toluene and n-heptane up to 2600 bar, Physics and Chemistry of Liquids 14 (4), 273-296 (1985); doi: 10.1080/00319108508080991 